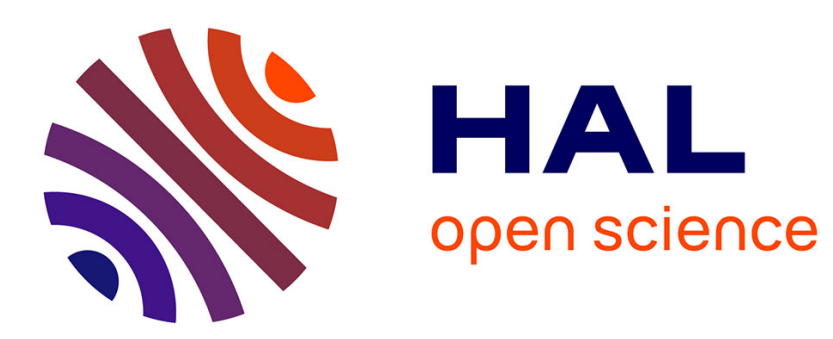

\title{
Incentives to patients versus incentives to health care providers: The users' perspective
}

\author{
Izabela Jelovac, Philippe Polomé
}

\section{To cite this version:}

Izabela Jelovac, Philippe Polomé. Incentives to patients versus incentives to health care providers: The users' perspective. 2015. halshs-01142578

\author{
HAL Id: halshs-01142578 \\ https://shs.hal.science/halshs-01142578 \\ Preprint submitted on 15 Apr 2015
}

HAL is a multi-disciplinary open access archive for the deposit and dissemination of scientific research documents, whether they are published or not. The documents may come from teaching and research institutions in France or abroad, or from public or private research centers.
L'archive ouverte pluridisciplinaire HAL, est destinée au dépôt et à la diffusion de documents scientifiques de niveau recherche, publiés ou non, émanant des établissements d'enseignement et de recherche français ou étrangers, des laboratoires publics ou privés. 
UMR 5824

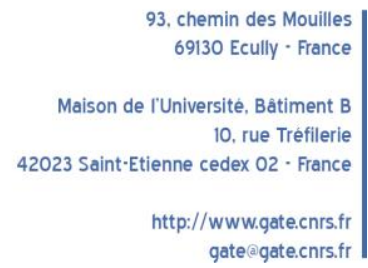

WP 1510 - April 2015

\title{
Incentives to patients versus incentives to health care providers: The users' perspective
}

\author{
Izabela Jelovac, Philippe Polomé
}

\begin{abstract}
:
In theory, health care providers may adapt their professional behavior to the financial incentives driven by their remuneration. Our research question is whether the users of health care services anticipate such a behavior from their general practitioner (GP) and, if they do, what are the consequences of such an anticipation on their preferences regarding financial incentives. We propose a theoretical model to identify potential determinants of such preferences. We empirically test our theoretical predictions using the data from a survey that elicits individual preferences for either patients' or providers' hypothetical incentives in France. The empirical results confirm the theoretical ones by establishing that users tend to prefer to pay a copayment themselves when the amount of GPs' incentives is high, the one of the patients' copayment is low, they anticipate that their GP's medical decisions are affected by financial incentives and their wealth is high. Otherwise, they prefer their GP to face financial incentives.
\end{abstract}

Keywords:

incentives, health care providers, patients, individual preferences

JEL codes:

I13, I18, J33

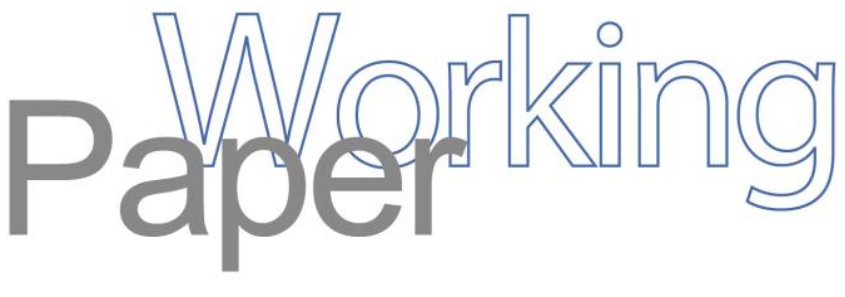




\title{
Incentives to patients versus incentives to health care providers: The users' perspective*
}

April 2015

\author{
Izabela Jelovac ${ }^{\dagger}$ \\ Philippe Polomé
}

\begin{abstract}
In theory, health care providers may adapt their professional behavior to the financial incentives driven by their remuneration. Our research question is whether the users of health care services anticipate such a behavior from their general practitioner (GP) and, if they do, what are the consequences of such an anticipation on their preferences regarding financial incentives. We propose a theoretical model to identify potential determinants of such preferences. We empirically test our theoretical predictions using the data from a survey that elicits individual preferences for either patients' or providers' hypothetical incentives in France. The empirical results confirm the theoretical ones by establishing that users tend to prefer to pay a copayment themselves when the amount of GPs' incentives is high, the one of the patients' copayment is low, they anticipate that their GP's medical decisions are affected by financial incentives and their wealth is high. Otherwise, they prefer their GP to face financial incentives.
\end{abstract}

JEL codes: I13, I18, J33

Keywords: incentives, health care providers, patients, individual preferences

* The authors thank Luigi Siciliani, Bruno Ventelou, Peter Zweifel, participants to the $13^{\text {th }}$ European Health Economics Workshop in Seville and seminar participants at Gate Lyon-St Etienne, Swiss School of Public Health, University of Burgundy and University of Vigo. Financial support from IReSP and from Chaire Santé is gratefully acknowledged.

$\dagger$ University of Lyon, Lyon, F-69007, France; CNRS, GATE Lyon Saint-Etienne, Ecully, F-69130, France. jelovac@gate.cnrs.fr

† University of Lyon, Lyon, F-69007, France; CNRS, GATE Lyon Saint-Etienne, Ecully, F-69130, France; University Lyon 2, Lyon, F-69007, France.polome@gate.cnrs.fr 


\section{Introduction}

In theory, health care providers may adapt their professional behavior to the incentives they face. Our research question is whether the users of the health care system anticipate such a behavior from their GP and, if they do, what are the consequences of such an anticipation on their preferences regarding financial incentives in health care.

Health authorities in many countries increasingly use incentives to control the raising health care expenditure. They do this by imposing health-care costs sharing onto patients (demandside cost sharing) and/or onto health care providers (supply-side cost sharing). Concretely, cost-containment incentives to patients take the form of insurance copayments or deductibles. In turn, cost-containment incentives to health care providers can be associated with their remuneration. Remuneration mechanisms such as capitation or, more generally, prospective budgets incorporate such incentives since health care providers become residual claimants on health care costs when they prescribe or provide treatments. ${ }^{1}$

The existing literature about optimal incentives in health care points to the trade off between alternative problems. On the one hand, patients' incentives are associated with financial risk and restricted access to health care. On the other hand, providers' incentives are generally associated with quality deterioration and selection by the providers of the less expensive patients. $^{2}$ This basic trade off has been nuanced in different ways. ${ }^{3}$ All together, health economists have established a quite complete set of pros and cons for both types of incentives. However, there is no accurate indication so far as to whether the very users of health care services prefer one or another incentives scheme, should they choose one among them.

The experience with incentives in health care in the US is useful to motivate our research question. Most health insurance plans before the 1980s were conventional: Insurers reimbursed health care providers for each health service provided (physicians were paid feefor-service), using patients' copayments and deductibles to provide incentives against

\footnotetext{
Capitation in this context means a set amount for each enrolled person assigned to one health care provider or group of health care providers, whether or not that person seeks care.

2 See Ellis and McGuire (1990); Ellis and McGuire (1993).

3 See Jelovac (2014) for a summary.
} 
excessive utilization of health care services. ${ }^{4}$ In the late 1980s and early 1990s, managed care organizations became dominant in the US. Managed care organizations traditionally use a variety of techniques intended to reduce the health care spending, among which financial incentives for health care providers (capitated reimbursement for each patient regardless of cost, for example). The price of insurance was typically lower for patients. Managed care has been heavily criticized in the public arena at the end of the 1990s because of the perceived low quality of health care. As a response to this managed care backlash, state governments have passed the "patients' bills of rights" to restrict the use of providers' incentives and other cost-containment techniques.

In the EU countries, the public sector is traditionally more involved in the organization of the health care system than in the US. In social insurance systems in the EU (Belgium, France, Germany, Luxembourg), fee-for-service arrangements tend to be the predominant payment type in both general practice and specialist care out-of-hospital. The users of the health care system are insured either by the public health insurance or within a regulated private health insurance sector. The insurance coverage has generally been generous until recently in the EU. In the recent decades, many EU governments have either introduced or increased the levels of patients' copayments to control the growing health care spending. Incentives to health care providers are far less widespread in the EU than in the US. Some governments consider such providers' incentives despite the generally active lobbying of physicians against incentives. In France, the Direction générale du Trésor (2008) claims that diversity in providers' payment schemes may be useful to improve the quality of the medical activity. ${ }^{5}$ Such a choice of payment system by providers may result in self-selection by both patients and providers according to wealth, health and quality dimensions, in particular if some payment systems include providers' incentives and others not. ${ }^{6}$

To anticipate the effects of hypothetical new incentives in the health care sector and to avoid any adverse public reaction such as the US managed care backlash, it is important to gather information about the users' preferences regarding financial incentives. So far, such preferences between patients' incentives and providers' incentives are not documented. We

\footnotetext{
See Pinkovskiy (2014).

5 Direction générale du Trésor is the department of the French Ministry of Finance that is in charge of national funds and the financial and economic system.

$6 \quad$ See Allard et al. (2014); Jelovac (2014).
} 
observe that, quite rationally, the users of health care services resist increases in co-payments while providers resist financial incentives in their remuneration. However, the users' preferences can be ambiguous, should they choose one option between patients' and providers' incentives. If the users of health care services anticipate no relationship between their providers' remuneration and behavior, there is no reason for the users to prefer patients' incentives over providers' incentives. Indeed, users would not associate any quality limitation with providers' incentives. Roughly speaking, the only individual choice would be between paying a copayment in case of patients' incentives and paying nothing in case of providers' incentives. Conversely, if users anticipate a limitation in quality due to providers' incentives, they may trade off a financial loss from paying a copayment against a quality loss if the providers face incentives.

We propose in this paper both a theoretical and an empirical analysis of this issue. The theoretical model identifies potential determinants of the preferences for mutually exclusive incentives arrangements. It establishes the following intuitive relationships between preferences for any incentives scheme and the exogenous variables of the model. In theory, users prefer the providers' incentives if the amount of providers' incentives is low enough, their perceived providers' altruism is high enough, the co-payment (in the alternative of patients' incentives) is high enough, and their wealth is low enough. Otherwise, they prefer to face financial incentives themselves. The effect of the health status is ambiguous in theory.

We empirically test our predictions using the data from a survey using the discrete choice method to elicit people's preferences for either patients' incentives or providers' incentives. We perform a survey in the French general population (about 1000 individuals). On top of the results of the discrete choice method, the survey asks questions about the GP-patient relationship, risk attitudes (both financial and health related), health status and behavior, health insurance arrangements and socio-economic variables. The survey has been run in December 2011 by CSA. ${ }^{7}$

The health care system in France is characterized by public health insurance with partial reimbursement of health care expenses. Most individuals top up this partial public insurance with a private voluntary complementary health insurance. French health care providers are

7 http://www.csa.eu/en/n752/about-us.aspx 
paid fee-for-service for out-of-hospital care and some of them (although few GPs) are allowed to balance bill. The French general population is well acquainted with demand-side cost sharing mechanisms since a system of franchise has been introduced in France in 2005. This mechanism imposes a copayment for buying drugs, which cannot be reimbursed by any complementary insurance unless exempted because of health or social motives. This copayment amounts to $0.5 €$ per pack of prescription drugs. However, people in France are not familiar with providers' incentives since no such mechanism has been used for French physicians until recently. ${ }^{8}$ Therefore, an effort has been made to construct a simple providers' incentives scheme that mirrors the French franchise for buying pharmaceuticals. In the discrete choice method questions, we ask respondents to chose between either a "patient's copayment $p$ " for buying a drug or a "GP's copayment $m$ " for prescribing the same drug. The idea of a "GP's copayment" is based on a 1991 reform in the UK. According to this reform, a drugs-specific budget has been added to the GPs' revenue for GPs to pay for the drugs they prescribe.

The empirical results confirm the theoretical ones by establishing the following. First, for the levels of copayments that we consider, preferences between patients' incentives and GPs' incentives are mixed. Furthermore, as predicted by the theoretical model, the amounts of incentives play a central role. Respondents tend to prefer patients' incentives over providers' incentives the lower the amount of patients' incentives and the higher the amount of providers' incentives. The role of anticipations appears very clearly as well. Respondents tend to prefer patients' incentives over providers' incentives when they think that an increase in patients' copayment would not impact patients' health while a providers' copayment would. Our results also suggest that respondents tend to prefer patients' incentives when they own real estate, which is a proxy for wealth. Moreover, female respondents and respondents in good health tend to prefer patients' incentives over providers' incentives, conversely to male respondents.

The paper is organized as follows. Section 2 provides the theoretical analysis and its results. Section 3 describes the data set. Section 4 presents the empirical analysis and its results. Section 5 concludes.

8 GPs in France can earn a financial bonus if they satisfy a series of guidelines, among which one relates to cost-containment: the prescription of generic or less expensive drugs. This bonus scheme has been generalized to all GPs in France in January 2012, i.e., the month following our survey. 


\section{Theoretical model}

We assume that a representative patient wishes to consume the quantity of health care services $q^{D}$ that maximizes his total utility under a budget constraint:

$$
\operatorname{Max}_{\{q\}} V(h, q)+U(z) \quad \text { s.t. } \quad z+a q=I .
$$

The function $V($ ) reflects the utility from health, $q$ is the quantity of health care and $h$ is the initial health status (a healthy individual has a high $h$ ). To be coherent with the empirical analysis, we consider that the quantity of health care is actually the quantity of prescription drugs and that the health care provider is the general practitioner (GP). The function $U$ ( ) is the utility from the consumption of a composite good $z$, the price of which is normalized to 1 . We assume that $V($ ) and $U()$ are strictly increasing in their arguments and concave. The patient's copayment per unit of drug is denoted $a$ and his income or wealth is denoted $I$. Therefore, the quantity of drugs $q^{D}$ that is preferred by the patient is the one that solves (1) and it is implicitly defined by the first-order condition (2):

$$
\frac{V_{q}^{\prime}\left(h, q^{D}\right)}{U^{\prime}\left(I-a q^{D}\right)}=a
$$

Applying the implicit function theorem to (2), we obtain a quantity of drugs $q^{D}$ that increases with the patient's wealth and decreases in the copayment $a$ and in the health condition $h$ :

$$
\frac{\partial q^{D}(I, a, h)}{\partial I}>0 ; \quad \frac{\partial q^{D}(I, a, h)}{\partial a}<0 ; \quad \frac{\partial q^{D}(I, a, h)}{\partial h}<0 .
$$

We further assume that the GP wishes to prescribe the quantity of drugs $q^{S}$ that maximizes her own total utility, which partly coincides with the patient's utility:

$$
\operatorname{Max}_{\{q\}} \beta V(h, q)+(1-\beta)(Y+B-b q) .
$$


The parameter $\beta$ represents the weight of the patient's health into the GP's total utility. Concretely, it reflects the GP's sense of professional duty or altruism. $Y$ is her basis revenue; $B$ is some drugs-specific prospective budget while $b$ is the share of the costs associated to the prescription of drugs that is borne by the GP, i.e., $b$ is the GP's copayment per unit of drug. ${ }^{9}$ Therefore, the quantity of drugs $q^{S}$ that the GP wishes to prescribe maximizes (4) and it satisfies the first-order condition (5):

$$
V_{q}^{\prime}\left(h, q^{s}\right)=b \frac{1-\beta}{\beta} .
$$

Applying the implicit function theorem to (5), we obtain that the quantity of drugs $q^{S}$ is increasing in the altruism parameter $\beta$, and it is decreasing in the GP's copayment $b$ and in the health status $h$ :

$$
\frac{\partial q^{S}(\beta, b, h)}{\partial \beta}>0 ; \quad \frac{\partial q^{S}(\beta, b, h)}{\partial b}<0 ; \quad \frac{\partial q^{S}(\beta, b, h)}{\partial h}<0 .
$$

We are actually interested in the perception of a possible link between the GP's behavior and her financial incentives. Therefore, the altruism parameter $\beta$ that is relevant here is the one perceived by the patient. A high $\beta$ corresponds to a patient's belief that his GP only cares about his health when prescribing drugs, thus ignoring any incentive effect of the copayment $b$. On the contrary, a low $\beta$ implies that the patient anticipates a relationship between his GP's behavior and remuneration. Formally, it is easy to show that the incentive power of $b$ is decreasing in $\beta$ and it tends to be nul when $\beta$ tends to 1 :

$$
\frac{\partial^{2} q^{S}(\beta, b, h)}{\partial b \partial \beta}<0 \quad \text { and } \quad \frac{\partial q^{S}(\beta, b, h)}{\partial b} \rightarrow 0 \text { when } \beta \rightarrow 1 .
$$

As in Ellis and McGuire (1990), we assume that the short side of the market dominates if there is a divergence between demand $q^{D}$ and supply $q^{S}$. That is, the GP prescribes the lowest

9 A more general model would consider $q$ as the quantity or intensity of any medical service. It would also explicitly include a fee-for-service payment for the GP. Denoting this fee $f$, we would write the GP's total utility as $b V(h, q)+(1-b)(Y+f q+B-b q)$. In this case, the GP is a residual claimant when providing medical services only if $b>f$. Our model simplifies this approach without any loss of generality. Our parameter $b$ simply replaces the difference $b-f$ in the more general version of the model. 
of both quantities. If the GP alone pays a copayment ( $b>0$ et $a=0$ ), she prescribes a quantity $q^{S}$ that is lower than $q^{D}$. Conversely, if the patient alone pays a copayment $(b=0$ et $a>0)$, the GP prescribes the quantity $q^{D}$ that is preferred by the patient and it is lower than $q^{S}$.

The relevant question now is whether the patient prefers either to pay a copayment $a$ and get a quantity of drugs $q^{D}$ or to have his GP pay a copayment $b$ and get the quantity of drugs $q^{S} .{ }^{10}$ This is equivalent to sign the difference in patient's utilities between the two scenarios. Therefore, we define the function $\Delta$ as follows:

$$
\begin{aligned}
\Delta(I, a, \beta, b, h)=\quad & {\left[V\left(h, q^{S}(\beta, b, h)\right)+U(I)\right] } \\
- & {\left[V\left(h, q^{D}(I, a, h)\right)+U\left(I-a q^{D}(I, a, h)\right)\right] . }
\end{aligned}
$$

If $\Delta(I, a, \beta, b, h)$ is positive, the patient prefers his GP to pay the copayment $b$. If it is negative, he prefers to pay a copayment $a$ himself. We cannot strictly sign $\Delta$ without making further unrealistic assumptions. However, we can analyze the influence of our parameters on this difference in utilities, $\Delta$. Using the relationships (2) and (5) defining $q^{S}$ and $q^{D}$, respectively, and differentiating $\Delta(I, a, \beta, b, h)$ totally in (8), we obtain the following relationships:

$$
\begin{aligned}
& \frac{\partial \Delta}{\partial b}<0 ; \quad \frac{\partial \Delta}{\partial \beta}>0 ; \quad \frac{\partial \Delta}{\partial a}>0 ; \quad \frac{\partial \Delta}{\partial I}>0 ; \\
& \text { and either } \quad \frac{\partial \Delta}{\partial h}>0 \quad \text { or } \quad \frac{\partial \Delta}{\partial h}<0 .
\end{aligned}
$$

These relationships imply that a patient prefers to pay a copayment himself rather than his GP when the amount of the GP's copayment that is considered is high enough, the one of the patient's copayment is low enough, the patient has a low perception of his GP's altruism and the patient's wealth is high enough. Otherwise, a patient prefers providers' incentives. All these relationships are intuitive.

10 We only consider the choice between mutually exclusive incentive schemes. Our idea is that combining patients' incentives and providers' incentives would only allow to sum the disadvantages of both incentives schemes without summing their advantages. Indeed, one incentives scheme is sufficient to limit the quantity of health care that is prescribed and consumed when it coincides with the short side of the market. 
Furthermore, the effect of the health condition on a patient's preferences is ambiguous. Depending on whether the GP's copayment is high or low relative to the patient's copayment, the GP prefers to prescribe a quantity of drugs that is higher or lower relative to the one preferred by the patient if the latter had to pay the copayment. Therefore, the patient's preferences are not well defined as a function of their health.

The following proposition summarizes these theoretical findings.

\section{Proposition 1.}

When patients' incentives $a$ and GPs' incentives $b$ are mutually exclusive, a representative user prefers patients' incentives a over GPs' incentives $b$ if

- the amount of the GPs' incentives $b$ is relatively high;

- the amount of the patients' incentives a is relatively low;

- the GP's altruism that is perceived by the representative user is relatively low;

- the representative user's wealth is relatively high.

Otherwise, the representative user prefers GPs' incentives b over patients' incentives $a$. The effect of the health status on such preferences is ambiguous.

\section{Data}

Opinion poll company CSA administered a jointly designed survey in December 2011 in the general population aged 18-60 in France. ${ }^{11}$ There were 1008 respondents. The questionnaire had been tested in focus groups and pilot studies, including monitoring of pilot subjects with debriefing. The questionnaire was administered in face-to-face interviews lasting about three quarter of an hour.

\section{The dependent variable}

To construct our dependent variable "Probability to prefer a patients' copayment over a GPs' copayment", we use the discrete choice method in the following way. After reminding the users of the existence of the patients' copayment, and explaining them how a GPs' copayment

\footnotetext{
${ }^{11}$ http://www.csa.eu/en/n752/about-us.aspx
} 
would work, the users state their preference between a patients' copayment $a$ (scenario A) and a GPs' copayment $b$ (scenario B). The patients' copayment and the GPs' copayment are mutually exclusive: A positive patients' copayment $a$ associated with a zero GPs' copayment characterizes scenario A while a positive GPs' copayment $b$ associated with a zero patients' copayment characterizes scenario B. The amounts of the patients' copayment $a$ vary between $0.75 €$ and $2 €$ in scenario A while the amounts of the GPs' copayment $b$ vary from $2 €$ to $6 €$ in scenario B. The discrete choice question is asked three times, with varying amounts of $a$ and $b$. Table 1 illustrates a vignette with a discrete choice between scenario A and scenario $\mathrm{B}$.

Table 1. Discrete choice method.

\begin{tabular}{|l|c|c|}
\cline { 2 - 3 } \multicolumn{1}{c|}{} & GPs' ${ }^{1}$ incentives & Patients' ${ }^{1}$ incentives \\
\hline Scenario $A$ & 0 & $a$ \\
\hline Scenario $B$ & $b$ & 0 \\
\hline
\end{tabular}

The respondents answer either they prefer scenario A, or they prefer scenario B, or they are indifferent between both scenarios, or they do not want to answer. ${ }^{12}$ The following table provides descriptive statistics about the respondents' preferences.

Table 2. Descriptive statistics about the respondents' preferences.

\begin{tabular}{|l|l|l|l|}
\cline { 2 - 4 } \multicolumn{1}{c|}{} & $1^{\text {st }}$ iteration & $2^{\text {nd }}$ iteration & $3^{\text {rd }}$ iteration \\
\hline Prefer scenario A & $35 \%$ & $37 \%$ & $32 \%$ \\
\hline Prefer scenario B & $43 \%$ & $39 \%$ & $31 \%$ \\
\hline Are indifferent between A and B & $12 \%$ & $11 \%$ & $18 \%$ \\
\hline Do not respond & $10 \%$ & $13 \%$ & $19 \%$ \\
\hline
\end{tabular}

Both copayments $a$ and $b$ can take any among four possible amounts in Euros: $a \in\{0.75,1$, $1.5,2\}$ and $b \in\{2,3,4.5,6\}$. These amounts are unrelated to the public budget or social security budget, but have been devised using pilot studies and sequential design to induce

12 Our model is a discrete choice model, or qualitative choice model, in the sense that it describes, explains, and predicts choices between two discrete alternatives. Our aim is not to elicit a value for a willingness to pay, or to accept compensation for, one or another scenario. It has therefore no relationship with the so-called choice modeling techniques, which are specifically used to estimate the value of the willingness to pay or to accept through a choice experiment. 
respondents' reactions, that is, primarily for econometric reasons. ${ }^{13}$ To use the discrete choice method efficiently, we only use intermediate values of $a(a=1$ or 1.5$)$ and $b(b=3$ or, 4.5) when asking the respondents' preferences for the first time. Then, to avoid redundant information and to avoid loosing credibility in the interview, the amounts proposed in the second preferences question depend on the answer to the first one. Consider for example a first-time choice between $a=1.5$ in scenario A and $b=3$ in scenario B and that a respondent states a preference for $a=1.5$ in scenario A over $b=3$ in scenario B. In this case, we consider that any subsequent choice between $a \leq 1.5$ in scenario $\mathrm{A}$ and $b \geq 3$ in scenario $\mathrm{B}$ is redundant because a rational respondent should continue to prefer $a \leq 1.5$ in scenario A over $b \geq 3$ in scenario B and no useful information would be gathered. Therefore, in the second preferences question, we only offer non redundant choices. We impose the same restriction on copayments $a$ and $b$ in the third preferences question unless the limit of possible copayments is reached during the second preferences question. The limit would be $a=b=2$ in the example. This is why there are three preferences question for most respondents (911 out of 1008 respondents) and only two for the remaining 9.6\%. The next table illustrates this example. Obviously, such procedure induces a form of endogeneity in the econometric analysis since each amount depends on the answer to previous preferences question.

Table 3. An example of copayments selection.

\begin{tabular}{|l|l|l|l|l|}
\cline { 2 - 5 } \multicolumn{1}{l|}{} & $a=0.75 €$ & $a=1 €$ & $a=1.5 €$ & $a=2 €$ \\
\hline$b=2 €$ & & & & \\
\hline$b=3 €$ & redundant & redundant & $\boldsymbol{a}=\mathbf{1 . 5} €>\boldsymbol{b}=\mathbf{3} €$ & \\
\hline$b=4.5 €$ & redundant & redundant & redundant & \\
\hline$b=6 €$ & redundant & redundant & redundant & \\
\hline
\end{tabular}

The regressors

Additionally to the preferences question just described, the questionnaire also asks approximately 70 questions about the patient-GP relationship (number of visits, GP's behavior, quality and duration of the relationship, trust, etc.); the risk attitudes (both financial

13 The sequential design allowed the researchers to update the amounts during survey administration, by waves of 100 interviews. The intention is to avoid falling completely outside of respondents' trade-offs, e.g. when most respondents either reject or accept one scenario whatever the amounts quoted in the interview. 
and health related); health status and behavior (subjective health, sport, smoking, vaccines, etc.); health insurance arrangements (private supplementary insurance, gate-keeping GP, etc.); demographic and socio-economic variables (household characteristics, education, income, real estate, etc.). Most of these questions are inspired by Allonier et al. (2008), Arrondel et al. (2005) and Miraldo et al. (2013). The survey additionally asks questions about the anticipated effects of both patients' and GPs' incentives according to respondents. The following table offers some descriptive statistics about these anticipations.

Table 4. Descriptive statistics about the respondents' anticipations.

\begin{tabular}{|l|c|c|}
\cline { 2 - 3 } \multicolumn{1}{c|}{} & $\begin{array}{c}\text { An increase in patients' copayment } a \\
\text { would impact patients' health }\end{array}$ & $\begin{array}{c}\text { A GPs' copayment } b \text { would impact } \\
\text { patients' health }\end{array}$ \\
\hline Certainly & $20 \%$ & $20 \%$ \\
\hline Probably & $25 \%$ & $26 \%$ \\
\hline Probably not & $27 \%$ & $26 \%$ \\
\hline Certainly not & $25 \%$ & $21 \%$ \\
\hline Unsure / DK & $4 \%$ & $7 \%$ \\
\hline & A GPs' copayment $b$ would lead the GP & A GPs' copayment $b$ would lead the GP to \\
& to prescribe less & $11 \%$ \\
\hline Certainly & $20 \%$ & $23 \%$ \\
\hline Probably & $31 \%$ & $29 \%$ \\
\hline Probably not & $19 \%$ & $28 \%$ \\
\hline Certainly not & $17 \%$ & $9 \%$ \\
\hline Unsure / DK & $14 \%$ & delay prescriptions \\
\hline
\end{tabular}

\section{Empirical analysis}

\section{Empirical strategy}

The answers to the discrete choice questions used here are multinomial and multivariate. They are multinomial because respondents have the choice between four possible answers: Either 
they prefer scenario A, or they prefer scenario B, or they are indifferent between both scenarios, or they do not respond. They are multivariate because the respondents make this discrete choice three times. These are therefore referred to as three periods. Ideally, we would use a Multinomial Multivariate Probit model. To our knowledge, such a model has not been implemented so far. Hence, we use a Multivariate Probit model as in Cameron and Quiggin (1994). We motivate this choice in what follows.

We are interested in explaining the choice between scenarios $\mathrm{A}$ and $\mathrm{B}$, not the indifference between scenarios A and B or refusing to choose. Therefore, we use dichotomous data rather than multinomial data. We consider two strategies for transforming the multinomial data into dichotomous ones: Either we drop the data of those who are indifferent between A and B and those who do not answer, or we merge them with the answers of those who prefer B. So, our dichotomous data become either "prefer scenario A" versus "prefer scenario B", or "prefer scenario A" versus "do not prefer scenario A". Similar results between both strategies would indicate that selection bias is not an issue.

As noted in Section 3, we restrict the possible discrete choices in periods 2 and 3 to nonredundant choices. As a consequence, the discrete choice that we propose to a respondent in period 2 depends on his answer in period 1 and the discrete choice in period 3 depends on his answers in periods 1 and 2. This dependence between sequential choices and previous answers is an important source of endogeneity. To treat endogeneity, we follow Cameron and Quiggin (1994) and use Multivariate Probit. Concretely, we use FIML (full information maximum likelihood), following Cappellari and Jenkins (2003). ${ }^{14}$

Formally, the econometric specification goes as follows. Respondents state their preference between scenarios $\mathrm{A}$ and $\mathrm{B}$ in the first period. In scenario A, the respondent would pay a copayment $a_{1}$, while in scenario $\mathrm{B}$, the GP pays a copayment $b_{1}$. Thus, in terms of the following latent model where $c_{t}$ denotes copayment in period $t$ and $X$ denotes the timeinvariant individual characteristics:

14 An alternative strategy is to consider a Dichotomous Choice Panel Model with endogeneity, treating endogeneity by GMM similarly to Arellano and Bond (1991). However, the method of Cameron and Quiggin (1994) is more straightforward and does not require to define instruments. Moreover, a Dichotomous Choice Panel Model does not allow to analyze the coefficients of the regressors that do not vary between periods, which is very restrictive in our case. 


$$
y_{t}^{*}=\gamma c_{t}+X \delta+\varepsilon_{t}
$$

a respondent is asked to compare two utility levels in period 1 :

- utility in A with patients' copayment $a_{1}$ :

$$
y_{A 1}^{*}=\gamma_{A 1} a_{1}+X \delta_{A}+\varepsilon_{A 1}, \text { and }
$$

- utility in B with GPs' copayment $b_{1}$ :

$$
y_{B 1}^{*}=\gamma_{B 1} b_{1}+X \delta_{B}+\varepsilon_{B 1} \text {. }
$$

It is apparent that, in order to avoid that $X$ variables drop out of the choice entirely, it must be that they have different coefficients in the two choice situations $\mathrm{A}\left(a_{1}\right)$ and $\mathrm{B}\left(b_{1}\right)$. Further, the coefficient of the copayment amount cannot be restricted to be the same in the two choice situations since the two copayments do not have the same nature. Therefore, a respondent answers that he prefers $\mathrm{A}\left(a_{1}\right)$ over $\mathrm{B}\left(b_{1}\right)$ if

$$
y_{A 1}^{*}>y_{B 1}^{*}
$$

that is, if

$$
\gamma_{A 1} a_{1}+X \delta_{A}+\varepsilon_{A 1}>\gamma_{B 1} b_{1}+X \delta_{B}+\varepsilon_{B 1},
$$

or, if

$$
\varepsilon_{A 1}-\varepsilon_{B 1}<\gamma_{B 1} b_{1}-\gamma_{A 1} a_{1}+X \delta
$$

where $\delta=\delta_{A}-\delta_{B}$. Preference is written $A\left(a_{1}\right) \succ B\left(b_{1}\right)$ and indifferences are ruled out by assumption. Writing $z \sim n(0, \sigma)=g(z)$, the probability of the above event is thus

$$
\operatorname{Pr}\left\{A\left(a_{1}\right) \succ B\left(b_{1}\right)\right\}=\int_{-\infty}^{\left(\gamma_{B 1} b_{1}-\gamma_{A 1} a_{1}+X \delta\right) / \sigma} g(z) d z
$$

This is a conventional Probit model but for the fact that there are two amounts of copayments. There is a normalizing restriction that is often taken as $\sigma=1$, which is convenient here as this is also the one adopted in Cappellari and Jenkins (2003) Multivariate Probit procedure. 
The situation becomes more complex when two consecutive choice questions are asked to the respondents, and the copayments on the second question depend on the answer to the first question. However, this is formally similar to Double-bounded Contingent Valuation as treated by Cameron and Quiggin (1994) and thus we follow the same solution. Consider an original choice $\left[A\left(a_{1}\right), B\left(b_{1}\right)\right]$ and a follow-up $\left[A\left(a_{2}\right), B\left(b_{2}\right)\right]$. There are four cases:

- If $A\left(a_{1}\right) \succ B\left(b_{1}\right)$, then $a_{2} \geq a_{1}$ and $b_{2} \leq b_{1}$ (with at least one strict inequality) and

- $A\left(a_{2}\right) \succ B\left(b_{2}\right)$, or

- $A\left(a_{2}\right) \prec B\left(b_{2}\right)$.

- If $A\left(a_{1}\right) \prec B\left(b_{1}\right)$, then $a_{2} \leq a_{1}$ and $b_{2} \geq b_{1}$ (with at least one strict inequality) and

- $A\left(a_{2}\right) \succ B\left(b_{2}\right)$, or

- $A\left(a_{2}\right) \prec B\left(b_{2}\right)$.

Note that the follow-up $\left[A\left(a_{2}\right), B\left(b_{2}\right)\right]$ differs depending on the answer to the first question. We write $g\left(z_{1}, z_{2}\right)$ the Bivariate Normal density where $\left(z_{1}, z_{2}\right)$ is defined in a manner similar to $z$, but now we allow a covariance coefficient $\rho$ between the two random variables; the variances of both random variables are normalized to one as in the one-variable case. Following Cameron and Quiggin (1994), the probabilities associated with the previous event are therefore: ${ }^{15}$

- $\operatorname{Pr}\left\{\left[A\left(a_{1}\right) \succ B\left(b_{1}\right)\right]\right.$ and $\left.\left[A\left(a_{2}\right) \succ B\left(b_{2}\right)\right]\right\}=\int_{-\infty}^{\left(\gamma_{B 1} b_{1}-\gamma_{A 1} a_{1}+X \delta\right)} \int_{-\infty}^{\left(\gamma_{B 2} b_{2}-\gamma_{A 2} a_{2}+X \delta\right)} g\left(z_{1}, z_{2}\right) d z_{2} d z_{1}=P_{A A}$;

- $\operatorname{Pr}\left\{\left[A\left(a_{1}\right) \succ B\left(b_{1}\right)\right]\right.$ and $\left.\left[A\left(a_{2}\right) \prec B\left(b_{2}\right)\right]\right\}=\int_{-\infty}^{\left(\gamma_{B 1} b_{1}-\gamma_{A 1} a_{1}+X \delta\right)} \int_{\left(\gamma_{B 2} b_{2}-\gamma_{A 2} a_{2}+X \delta\right)}^{\infty} g\left(z_{1}, z_{2}\right) d z_{2} d z_{1}=P_{A B}$;

- $\operatorname{Pr}\left\{\left[A\left(a_{1}\right) \prec B\left(b_{1}\right)\right]\right.$ and $\left.\left[A\left(a_{2}\right) \succ B\left(b_{2}\right)\right]\right\}=\int_{\left(\gamma_{B 1} b_{1}-\gamma_{A 1} a_{1}+X \delta\right)}^{\infty} \int_{-\infty}^{\left(\gamma_{B 2} b_{2}-\gamma_{A 2} a_{2}+X \delta\right)} g\left(z_{1}, z_{2}\right) d z_{2} d z_{1}=P_{B A}$;

- $\operatorname{Pr}\left\{\left[A\left(a_{1}\right) \prec B\left(b_{1}\right)\right]\right.$ and $\left.\left[A\left(a_{2}\right) \prec B\left(b_{2}\right)\right]\right\}=\int_{\left(\gamma_{B 1} b_{1}-\gamma_{A 1} a_{1}+X \delta\right)}^{\infty} \int_{\left(\gamma_{B 2} b_{2}-\gamma_{A 2} a_{2}+X \delta\right)}^{\infty} g\left(z_{1}, z_{2}\right) d z_{2} d z_{1}=P_{B B}$.

15 Except that we impose that the coefficients of the $X$ variables remain the same in all choice situations. 
We define $l_{1}=1$ when $A\left(a_{1}\right) \succ B\left(b_{1}\right)$ and zero otherwise, and $l_{2}=1$ when $A\left(a_{2}\right) \succ B\left(b_{2}\right)$ and zero otherwise. The likelihood function can then be written using these probabilities as

$$
\mathcal{L}=\sum_{i} P_{A A}^{l_{1} l_{2}} P_{A B}^{l_{1}\left(1-l_{2}\right)} P_{B A}^{\left(1-l_{1}\right) l_{2}} P_{B B}^{\left(1-l_{1}\right)\left(1-l_{2}\right)}
$$

where the subscript $i$ refers to individual respondents. This essentially corresponds to the likelihood function implemented in Cappellari and Jenkins (2003) Multivariate Probit procedure.

As noted in Section 3, the third period data have some missing values (less than $10 \%$ ). Therefore, we run regressions both for the bivariate case (only periods 1 and 2 with 1008 respondents) and for the trivariate case (all three periods with 911 respondents).

For coherence with our theoretical model, we restrict the coefficients of the model to be identical across periods, except for the coefficients of the amount of copayments $a$ and $b$. We run an additional regression adding as regressors the answers to the previous bids, when available. That is, period 1 choice is used as additional regressor for period 2 choice, and both choices from periods 1 and 2 are used as additional regressors for period 3 choice. Similar results between both regressions would point to a kind of Granger causality of the copayments (and the individual characteristics X) to the choice between the scenarios A and B. In other words, copayments would add information above and beyond the previous period choice(s) to explain the current period choice, that is, respondents react to changing copayments.

Taking all these considerations into account, we end up presenting four regressions in Table 5. In both the trivariate regression TRIVmp and the bivariate regression BIVmp, the dichotomous dependent variable is "prefer scenario A" versus "do not prefer scenario A" and the regressors include answers to previous choices when available. In the trivariate regression TRIVm, the dichotomous dependent variable is "prefer scenario A" versus "do not prefer scenario A" and the regressors do not include answers to previous choices. In the trivariate regression TRIVsp, the dichotomous dependent variable is "prefer scenario A" versus "prefer scenario B" and the regressors include answers to previous choices when available. 


\section{Empirical results}

The descriptive statistics in Table 2 show a first interesting result: For the levels of copayments $a$ and $b$ that we consider, preferences between patients' incentives and GPs' incentives are mixed. Up to our knowledge, this result has not been reported in the literature so far. It may be an indication that some respondents do anticipate some link between their GP's incentives and medical decisions.

Table 5 displays the results of the four regressions related to the discrete choice answers. The four regressions lead to similar results and the estimated coefficient of our variables of interest are significantly different from zero. In particular, adding answers to previous choices to the set of regressors leads to very significant results without affecting the coefficients and significance of the other regressors. This can be interpreted in a Granger causal sense as mentioned earlier: People do not behave in a systematic way since changing copayments add something to the analysis.

\section{[Insert Table 5 about here]}

As predicted by the theoretical model, the amounts of incentives play a central role. The regressor "Amount $a$ " stands for the amount of patients" copayment while the regressor "Amount $b$ " stands for the amount of providers' copayment, both presented in the discrete choice questions. All four regressions in Table 5 suggest that respondents tend to prefer patients' incentives over providers' incentives the lower the amount of patients' incentives and the higher the amount of providers' incentives.

The role of anticipations appears very clearly as well. The regressor "Anticipated effect of $a$ " is a dichotomous variable that is constructed as follows. It is equal to 1 when respondents answer either "Certainly" or "Probably" to the question "Do you think that an increase in patients' copayment would impact patients' health?". It is equal to 0 when the answer to this question is either "Probably not" or "Certainly not". The regressor "Anticipated effect of $b$ " is constructed in a similar way, the only difference being the question: "Do you think that a providers' copayment would impact patients' health?". Regressions in Table 5 suggest that respondents tend to prefer patients' incentives over providers' incentives when they think that 
an increase in patients' copayment would not impact patients' health while a providers' copayment would. This empirical result about the anticipated effect on health of a providers' copayment parallels our theoretical result on perceived altruism: If the respondent perceives his GP to be very altruistic, he should not expect any effect on the GP's medical decisions (see Equation (7)) and, thereby, on his health.

The estimates of the regressor that illustrates respondents' wealth - "Real estate" - also appears to be significant in most regressions. This dichotomous regressor takes value 1 when the respondent owns no real estate. So, the results in Table 5 suggest that respondents tend to prefer patients' incentives when they own real estate. This confirms our theoretical result about the effect on users' wealth.

Gender appears to have estimates significantly different from zero as well. Table 5 suggests the following gender effect: Female respondents tend to prefer patients' incentives over providers' incentives, conversely to male respondents.

Two regressors related to health status - "Drug consumption" and "Subjective health" - are significant. "Drug consumption" provides information about the quantity of drugs prescribed during the last GP's consultation. "Subjective health" is a dichotomous variable with value 1 when the respondent has not felt hampered because of his health during the last 12 months. The results in Table 5 suggest that respondents in better health tend to prefer patients' incentives over providers' ones.

Last, the regressor "Foregoing healthcare" that illustrates whether or not the respondents have foregone the consumption of some prescribed drugs because of financial reasons, is not significant. So is the case for the regressor "Complementary insurance", which provides information about whether or not respondents are privately insured against reimbursable copayments. According to numerous trials not reported here, the data collected about risk attitudes and about relationships with GPs do not help explain the preferences for one or another incentive arrangement. 


\section{Conclusion}

To anticipate the effects of hypothetical new incentives in the health care sector and to avoid any adverse public reaction such as the US managed care backlash, it is important to gather information about the users' preferences regarding financial incentives. With this purpose in mind, we propose both a theoretical model and an empirical analysis to document the preferences of the users of the health care system between financial incentives to patients and financial incentives to providers. The empirical analysis uses data collected during a survey administered professionally in December 2011. In particular, the survey elicits the respondents' preferences for either providers' incentives or patients' incentives using a discrete choice model.

The theoretical model establishes that a representative user of health services tend to prefer providers' incentives if he perceives that his GP is altruistic enough, the amount of providers' incentives is low enough, the co-payment (in the alternative of patients' incentives) is high enough, and their wealth is low enough. Otherwise, he prefers to face financial incentives himself. The effect of the health status is ambiguous in theory.

The empirical analysis confirm the theoretical results. First, for the levels of copayments that we consider, preferences between patients' incentives and GPs' incentives are mixed. Concretely, not everyone prefers others, i.e. their GP, to pay. Furthermore, as predicted by the theoretical model, the amounts of incentives play a central role. Respondents tend to prefer patients' incentives over providers' incentives the lower the amount of patients' incentives and the higher the amount of providers' incentives. The role of anticipations appears very clearly as well. Respondents tend to prefer patients' incentives over providers' incentives when they think that an increase in patients' copayment would not impact patients' health while a providers' copayment would. Our results also suggest that respondents tend to prefer patients' incentives when they own real estate, which is a proxy for wealth. Moreover, female respondents and respondents in good health tend to prefer patients' incentives over providers' incentives, conversely to male respondents. 


\section{References}

Allard M, Jelovac I, Léger PT. 2014. Payment mechanism and GP self-selection: Capitation versus fee for service. International Journal of Health Care Finance and Economics 14(2): 143-160.

Allonier C, Dourgnon P, Rochereau T. 2008. Enquête sur la Santé et la Protection Sociale 2006. Rapport $n^{\circ}$ 1701. IRDES, Paris.

Arellano M, Bond S. 1991. Some tests of specification for panel data: Monte Carlo evidence and an application to employment equations. The review of Economic Studies 58: 277-297.

Arrondel L, Masson A, Verger D. 2005. Préférences face au risque et à l'avenir. Revue Economique 56(2): 393-416.

Blakely S. 1998. The Backlash against Managed Care. Nation's Business. July 1998.

Cappellari L, Jenkins SP. 2003. Multivariate Probit regression using simulated maximum likelihood. The Stata Journal 3: 278-294.

Direction générale du Trésor (2008). Mode de rémuneration des médecins. www.tresor.economie.gouv.fr/file/326885

Cameron TA, Quiggin J. 1994. Estimation using contingent valuation data from a "Dichotomous choice with follow-up" questionnaire. Journal of Environmental Economics and Management 27 (3): 218-234.

Ellis RP, McGuire TG. 1990. Optimal Payment Systems for Health Services. Journal of Health Economics 9: 375-396.

Ellis RP, McGuire TG. 1993. Supply Side and Demand Side Cost Sharing in Health Care. Journal of Economic Perspectives 7(4): 135-151.

Jelovac I. 2014. Primary Care, Gatekeeping, and Incentives. In : Anthony J. Culyer (ed.), Encyclopedia of Health Economics, Vol 3. San Diego : Elsevier. pp. 142-145.

Miraldo M, Galizzi M, Stavropoulou C. 2013. In sickness but not in wealth: Field evidence on patients' risk preferences in the financial and health domain. Working paper 12 579, Imperial College London, London, Imperial College Business School.

Pinkovskiy ML. 2014. The impact of the political response to the managed care backlash on health care spending: Evidence from State regulation of managed care. 


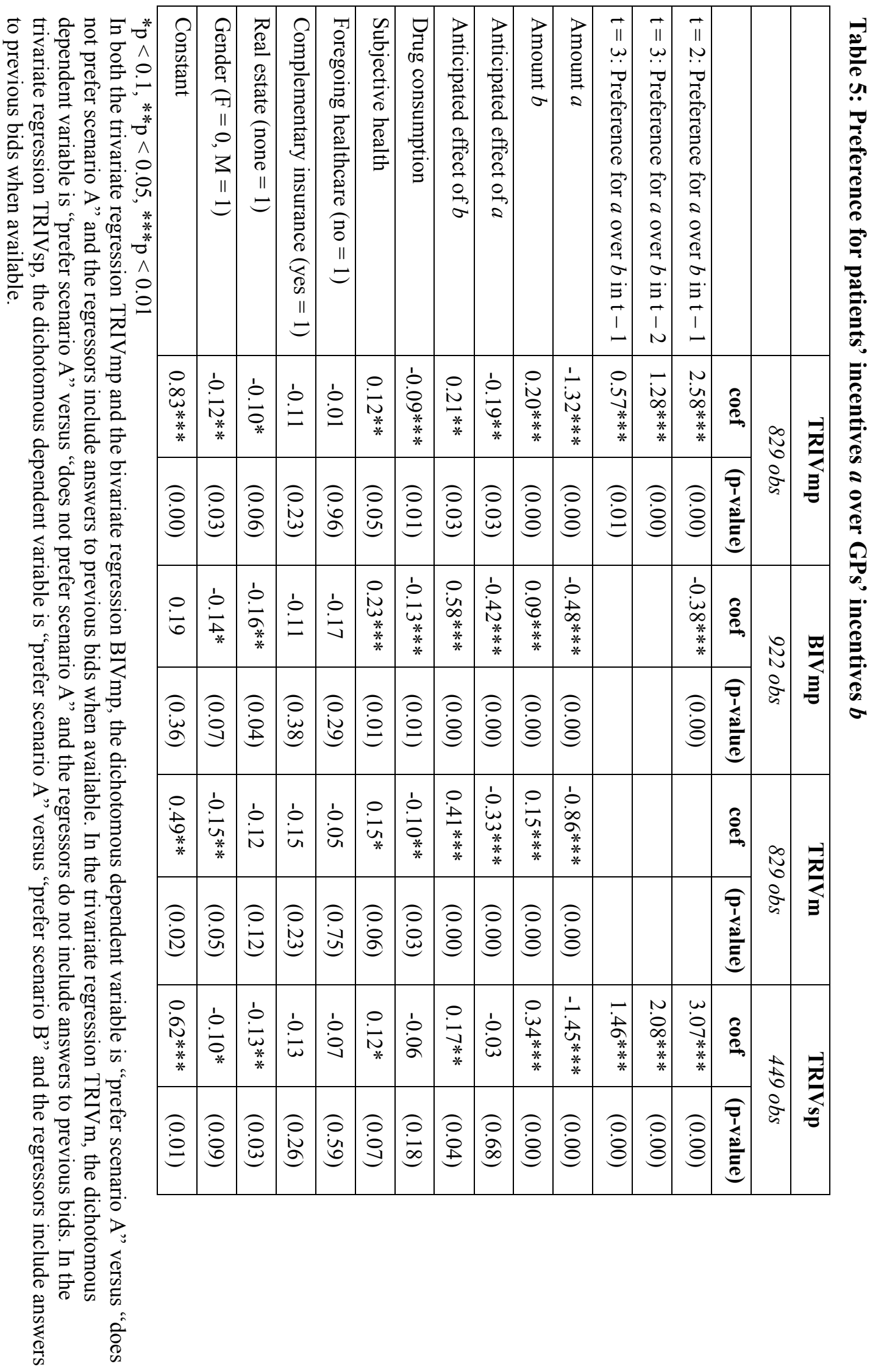

